\title{
Noise Generation Analysis of a Cylindrical Cavity by LES and Global Instability
}

\author{
Fabien Mery * \\ ONERA, Toulouse, France
}

\author{
Daniel C. Mincu ${ }^{\dagger}$ \\ ONERA, Chatillon, France \\ Alois Sengissen $§$ \\ Airbus, Toulouse, France
}

\author{
Gregoire Casalis $\ddagger$ \\ ONERA, Toulouse, France
}

\begin{abstract}
This paper is devoted to the computation of a compressible and high-Reynolds flow over a cylindrical cavity present on a flat plate. Two approaches are used : LES on one hand and linear stability analysis on the other one. The mean part of the former acts as input for the latter which consists in determining the corresponding eigenmodes. The results are compared to the unsteady part of the LES. The most amplified frequency in the LES seems to correspond to an eigenmode and a good agreement is also obtained when comparing the perturbed flow field.
\end{abstract}

\section{Introduction}

Several tests showed the occurrence of a tone generated by circular vent holes on the pressure side of a wing during approach conditions (Mach numbers ranging from 0.1 to 0.3$).{ }^{1}$ The French AEROCAV project aims to study the whistle, in order to understand the mechanisms at work in this geometry, and to propose a method to reduce this whistle by optimising the shape of the cavities. Among the different possible mechanisms, the intrinsic instability may be invoked has a candidate. Indeed, it is generally admitted that acoustic field is linked to shear flow instabilities for supersonic jets. ${ }^{2-4}$ In the cavity case, as the shear layer seems active in the noise generation, a linear stability analysis can also be useful to identify the eigenmodes and to determine their role in the noise generation. This paper presents results of a Large Eddy Simulation (LES) and a global linear stability approach of a flow over a cavity. The basic flow for the stability analysis corresponds to the mean part of the LES. As the flow is fully three-dimensional, the classical local stability theory is not appropriate. We must use the method initiated by Tatsumi ${ }^{5}$ and developed by Theofilis ${ }^{6}$ which can be called a global stability theory. Such stability analyses have been already and recently performed. ${ }^{7,8}$ However, these studies are limited to low Reynolds number (laminar flow). The present study deals with a high Reynolds number configuration inducing large differences comparing to the existing literature : the mean flow is compressible essentially turbulent and the stability analysis focuses only on the inviscid mechanisms. In the following part of the abstract, we provide first a description of the chosen configuration and of the global stability model. The main results are then reported.

\section{Modelisation}

\section{A. Description of the studied configuration}

The configuration retained for this study is a cylindrical cavity (see figure 1), with a diameter $D$ and a depth $H$ both equal to $0.1 \mathrm{~m}$. The inflow is an ideal gas with a constant specific heat, the freestream velocity is $U_{\infty}=70 \mathrm{~m} / \mathrm{s}$, corresponding to an inflow Mach number of $M=0.2$. The cavity diameter based Reynolds

\footnotetext{
*PhD student, ONERA-Department Modeles pour l'Aerodynamique et l'Energetique, Toulouse

$\dagger$ PhD student, ONERA-Department Simulation Numerique et Acoustique, Chatillon

¥Professor, ONERA-Department Modeles pour l'Aerodynamique et l'Energetique, Toulouse

$\S$ Engineer, Numerical Methods Group Acoustics and Environment, Airbus, France
} 
number is about 450000 . The upstream boundary layer is laminar and is about $15 \mathrm{~mm}$ thick at $0.2 \mathrm{~m}$ upward the cavity. The flow becomes rapidly turbulent from the upstream edge of the cavity.

The flow is computed by LES, using the ONERA in-house code called Flu3M. ${ }^{9}$ About 13 million mesh points with at least 40 points in the boundary layer are used. This simulation is in good agreement ${ }^{10}$ with experimental results. ${ }^{11}$ More details concerning the numerical procedure are available in the paper. ${ }^{10}$

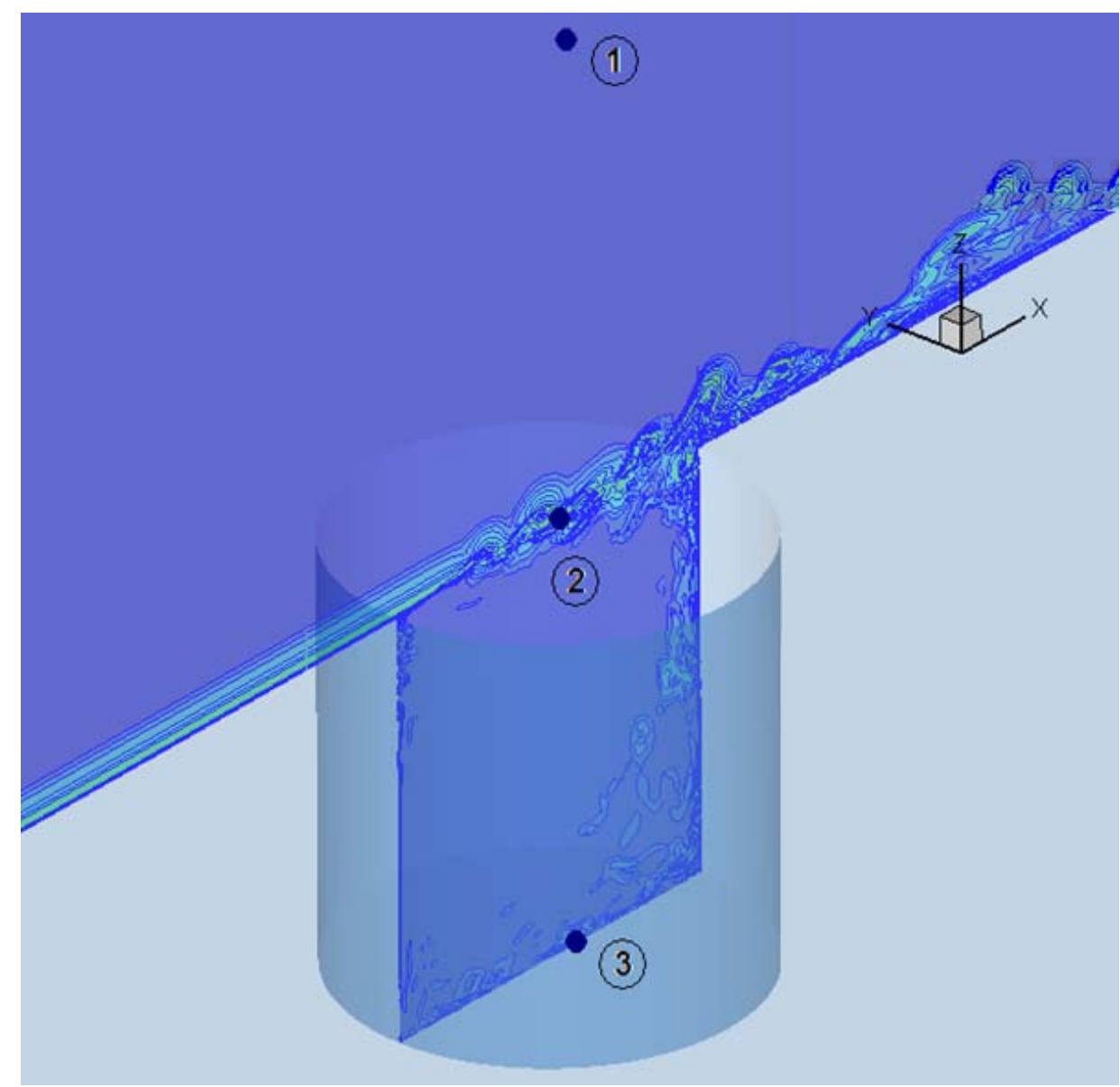

Figure 1. Studied configuration, sensors position and illustration of the instantaneous flow field in the median plane $(y=0)$.

For rectangular or circular cavities, Rossiter proposes a semi-empirical relationship which predicts dominant harmonics. ${ }^{12}$ Indeed, flow perturbations by impacting on downstream edge excite the shear layer and a particular tuning between the flow perturbations round-trip and the number of vortices in the shear layer may introduce self-sustained oscillations. By extension, the Rossiter relationship will be used in the present case. More precisely, we use a Rossiter relationship established by Block ${ }^{13}$ for low Mach numbers :

$$
S t_{\text {Rossiter }_{n}}=\frac{n}{M\left(1+\frac{0.514}{D / H}\right)+\frac{1}{\kappa(D / H)}}
$$

with $\kappa(D / H)$ an empirical parameter and $n$ an integer corresponding to the considered harmonic number.

\section{B. Global stability approach}

The mean flow is fully three-dimensional in and around the cavity. Indeed, such a mean flow depends on the three space coordinates, this would lead to a stability problem with amplitude functions depending on the same three space coordinates. Such a 3D stability problem requires memory resources which are not available today. Consequently, we propose to limit the analysis to the median plan $y=0$ where the mean 
flow is two-dimensional in the sense that the two velocity components $(\bar{u}(x, z), \bar{w}(x, z))$ are functions of $x$ and $z$ only, global stability theory can be applied.

To derive the stability equation, we neglect the mean pressure and the mean density gradients in accordance to the LES results. The stability is conducted using non-dimensionalized variables. To obtain dimensionless quantities (lengths, velocity components and time), we use the cavity diameter $D$, the free stream velocity $U_{\infty}$ and finally the ratio $D / U_{\infty}$. The stability studies are performed with the linearized Euler equations written for a compressible flow. Indeed, the goal of this study is to find a link between instabilities and the acoustic, so a compressible formulation is thus appropriate. Moreover, the mechanism of these instabilities lies on a velocity gradient in the shear layer ${ }^{14}$; viscous effects for the perturbations are assumed to play a minor role in these mechanisms ; an inviscid modeling is hoped enough to point out the unstable modes responsible for the acoustic. Table 1 summarizes the different assumptions done for the LES computation (mean flow and unsteady part) and for the stability analysis.

\begin{tabular}{c|c} 
LES computations & Stability problem \\
\hline viscous & inviscid \\
compressible & compressible \\
$3 \mathrm{D}$ & $2 \mathrm{D}, y=0$ \\
perfect gas, $C_{p}, C_{v}$ constant & perfect gas, $C_{p}, C_{v}$ constant \\
& $\bar{p}, \bar{\rho}$ constant \\
\hline
\end{tabular}

Table 1. Summary of the assumptions for the LES computations and the stability analysis

The Euler equation system is :

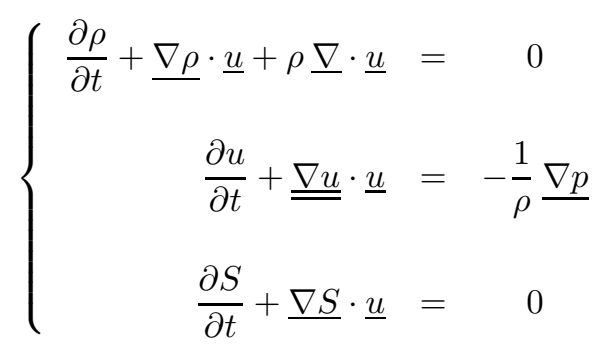

with $\rho$ the density, $p$ the pressure, $\underline{u}=(u, v, w)$ the velocity vector in the $(x, y, z)$ coordinates system and $S$ the entropy.

According to the linear stability theory (denoted by LST in the following), a systematic small perturbation is superposed to each mean flow quantity, i.e. each variable is decomposed into $q=\bar{q}+\widetilde{q}$ with $\bar{q}$ the mean part and $\widetilde{q}$ the perturbation part. Neglecting non linear terms and assuming that the mean quantities satisfy the equations, system (2) leads to system (3):

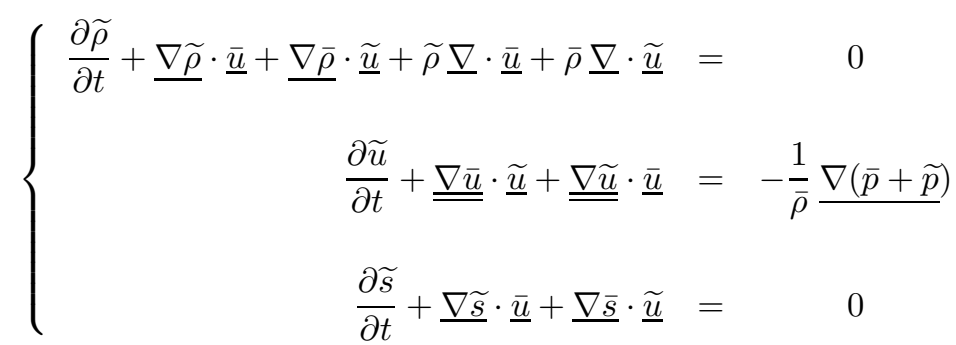

According to the assumptions presented in the previous section the transverse velocity perturbation is assumed to be zero and these three functions do not depend on $y$. Moreover, thanks to the nearly constant mean density and mean pressure, the entropy conservation equation and the equation of mass continuity can be simplified and so the system size can be reduced with only three independent functions : $\widetilde{u}, \widetilde{w}$ and $\widetilde{p}$. We consider the perturbations in the form of normal modes :

$$
\widetilde{q}(x, z, t)=\hat{q}(x, z) e^{-i \omega t}
$$


with $\hat{q}(x, z)$ a complex amplitude function and $\omega$ a complex number. The real part $\omega_{r}$ of $\omega$ corresponds to a circular frequency. We define an eigenmode Strouhal number as follows

$$
S t=\frac{\omega_{r}}{2 \pi}
$$

The imaginary part $\omega_{i}$ represents a temporal growth rate of the perturbation, a positive value means an amplified perturbation. The normal mode form equation (4) leads to an eigenvalue problem via a partial derivative equations system :

$$
\left\{\begin{aligned}
M^{2}\left(-i \omega \hat{p}+\bar{u} \hat{p}_{, x}+\bar{w} \hat{p}_{, z}\right)+\left(\hat{u}_{, x}+\hat{w}_{, z}\right) & =0 \\
\left(-i \omega \hat{u}+\bar{u}_{, x} \hat{u}+\bar{u}_{, z} \hat{w}+\bar{u} \hat{u}_{, x}+\bar{w} \hat{u}_{, z}\right)+\hat{p}_{, x} & =0 \\
\left(-i \omega \hat{w}+\bar{w}_{, x} \hat{u}+\bar{w}_{, z} \hat{w}+\bar{u} \hat{w}_{, x}+\bar{w} \hat{w}_{, z}\right)+\hat{p}_{, z} & =0
\end{aligned}\right.
$$

with the notation $f_{, r}=\frac{\partial f}{\partial r}$. There are three unknowns : $\hat{u}, \hat{w}, \hat{p}$, the amplification functions for the streamwise and vertical fluctuating velocity components and for the fluctuating pressure. System (5) has been mathematically characterized : characteristics' method enables to prove that for a Mach number $M$ less than 1, there is no favorite direction for the information propagation. Thus, there is a priori no strong constraints for the numerical method to be chosen in order to solve the previous system.

For the boundary conditions, on rigid boundaries, the normal velocity perturbation must vanish. In addition, we impose the two fluctuating velocity components to vanish on the non-rigid boundaries.

Regarding the mean flow, the critical zone is the shear layer over the cavity where the strong velocity gradient triggers Kelvin-Helmholtz instabilities. ${ }^{14}$ The classical use of the spectral methods would thus require a multi domain formulation in both directions $x$ and $z$ which would lead finally to a very large number of collocation points. Conversely, the finite element method is a good choice for solving partial differential equations over complex geometries even if the accuracy will be not so good than the one using a spectral method. We use the FREEFEM++ software (see http://www.freefem.org) to perform these finite element computations. Previous system (5) is written in variational form and the discretization is achieved by a finite element space composed of P1 piecewise linear continuous finite elements plus bubble applied on triangles. The mesh is tight up to an average of 35 vertices in the shear layer zone. A typical example is shown on figure 2. System (5) is expressed as a generalized eigenvalue problem in the form of

$$
\mathbf{A} q=\omega \mathbf{B} q
$$

where $\mathbf{A}$ and $\mathbf{B}$ are matrices and $q=\{\hat{p}, \hat{u}, \hat{v}\}$ eigenfunction vector. Computing the whole eigenmode spectrum takes too much time. The Arnoldi's method ${ }^{15}$ is appropriate in the present case. Actually, we are using a shift and invert method and the algorithm tries to converge on eigenvalues close to the chosen target value.

In order to put the stress on the unstable physical modes, many computations are necessary to reveal the unstable modes that are expected. Though, it is possible to build a "pseudo-spectrum" by varying the shift values. Then, these computations are carried out for different mesh grids in order to identify physical modes which must be independent on the mesh refinement. Furthermore, several computations have been driven on different domain sizes (e.g. the domain height has been varied from 2 to 5 cavity diameters). The following results are nearly independent of the domain size and of the mesh refinement ${ }^{\mathrm{a}}$.

\section{Numerical results}

\section{A. Stability results}

Four amplified modes have been obtained : all of them can be called Kelvin-Helmholtz modes (KH mode) because they are mainly concentrated in the shear layer (see left part of figures 3 and 4 ). The numerical values of the Strouhal number for the four modes are reported in table A together with the four first Rossiter modes computed thanks to (1).

\footnotetext{
${ }^{a} \mathrm{~A}$ full convergence is much more difficult for partial differential equation eigenvalue problem than for the classical OrrSommerfeld problem. Furthermore, in the cavity shear layer problem and with the finite element method such a full convergence appears a real challenge. On the real part of the eigenvalue $\omega$ and on the complex eigenfunction, the relative errors are of the order of $1 \%$.
} 


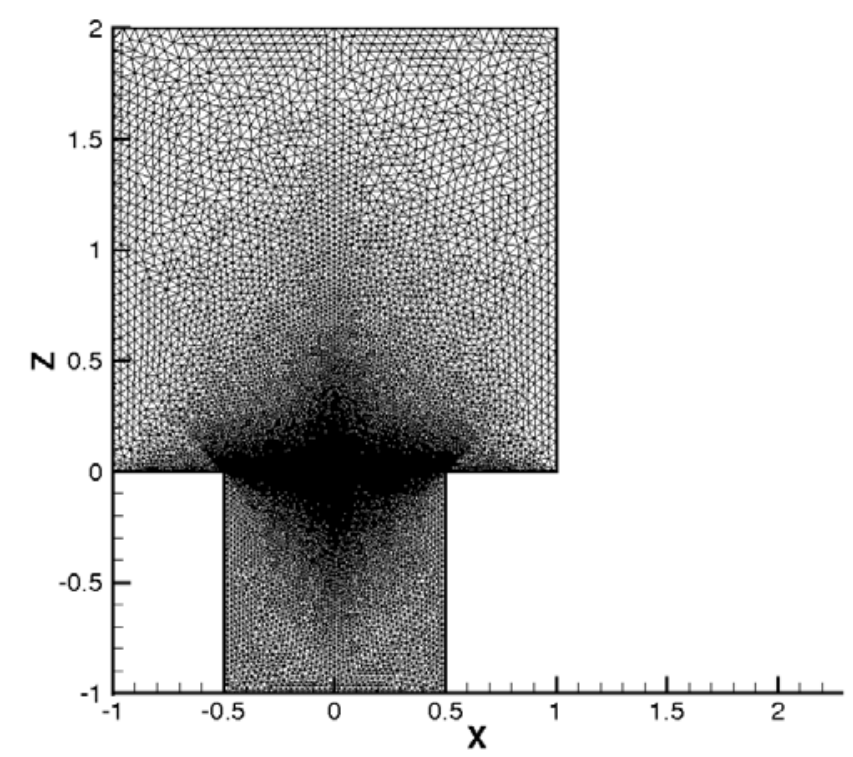

Figure 2. Mesh for the stability

\begin{tabular}{lcccc} 
& I & II & III & IV \\
\hline Strouhal number of KH mode & 0.51 & 0.93 & 1.28 & 1.55 \\
Strouhal number of Rossiter mode & 0.51 & 1.02 & 1.53 & 2.04
\end{tabular}

Table 2. Comparison between Strouhal numbers predicted by Equation (1) and the Strouhal numbers of Kelvin-Helmholtz modes

In table A, we can notice that for the three first Rossiter's modes, we are able to find corresponding values in the KH family. Indeed, Rossiter's mode I and II correspond to KH I and KH II, and Rossiter's mode III corresponds to KH IV. Originally, Rossiter's relationship expresses a special tuning between acoustic waves and shear layer instabilities whereas KH modes are only dependent on the mean flow. This study tends to show that the Rossiter self-sustained oscillations seem to be conditioned by the unstable Kelvin-Helmholtz modes. This consideration may be a first explanation of the origin of these special Rossiter's frequencies. We confirm this idea by analysing these modes in detail in the following. Figures 3 and 4 present the different shape of the $\mathrm{KH}$ modes thanks to their pressure modulus fluctuations. In KH I, it can be roughly counted one "structure", i.e. the wave length is about $\lambda_{K H I} / D=1$. In KH II, it can be roughly counted two "structures", i.e. the wave length is about $\lambda_{K H I I} / D=0.5$. Similarly, KH III has three "structures", i.e. a wave length $\lambda_{K H I I I} / D=0.33$ and $\mathrm{KH} \mathrm{IV}$, four "structures", i.e. $\lambda_{K H I V} / D=0.25$

Figure 5 shows a classical linear stability study on a 1D velocity profile (1D LST), the Blumen's modelisation $^{16}$ is used and the velocity profile is taken at the middle of the cavity.

Finally, KH I and KH II are in very good agreement with the 1D LST. We can conclude that the global stability theory takes into account the edge of the cavity : the wave length of the instabilities are forced by the cavity shape. The non parallel effects ( $x$-dependence of the mean flow) are often involved to justify a global stability analysis similar as the present one. In the present configuration, figure 5 seems to show that the global stability analysis acts essentially as a discretization of the continuous 1D curve ; the boundary conditions in $x$ written for the perturbation play a major role in this discretization process. 


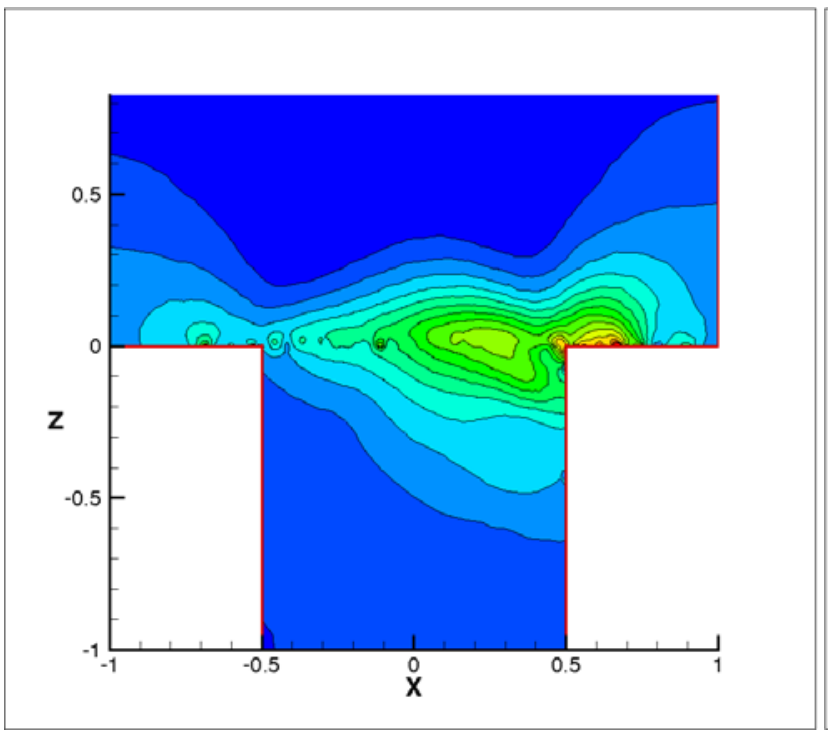

(a) $\mathrm{KH} \mathrm{I}$

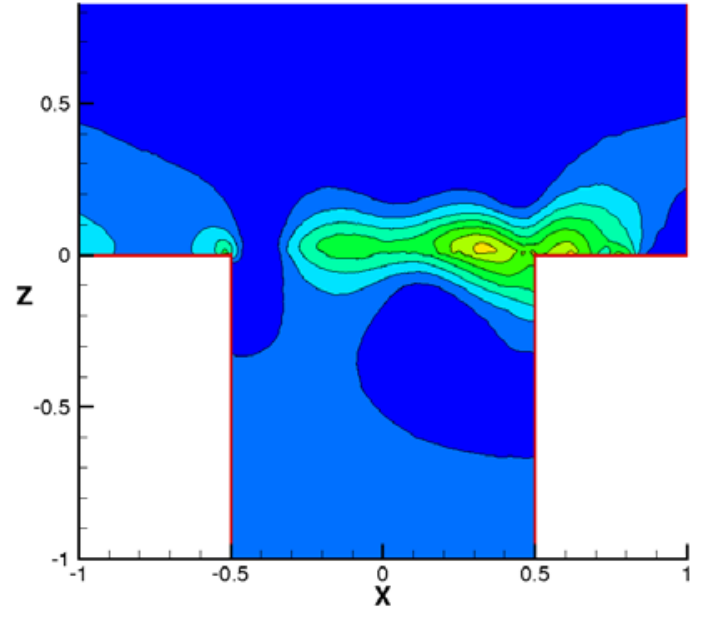

(b) KH II

Figure 3. KH modes I and II. Iso-values of the pressure perturbation modulus

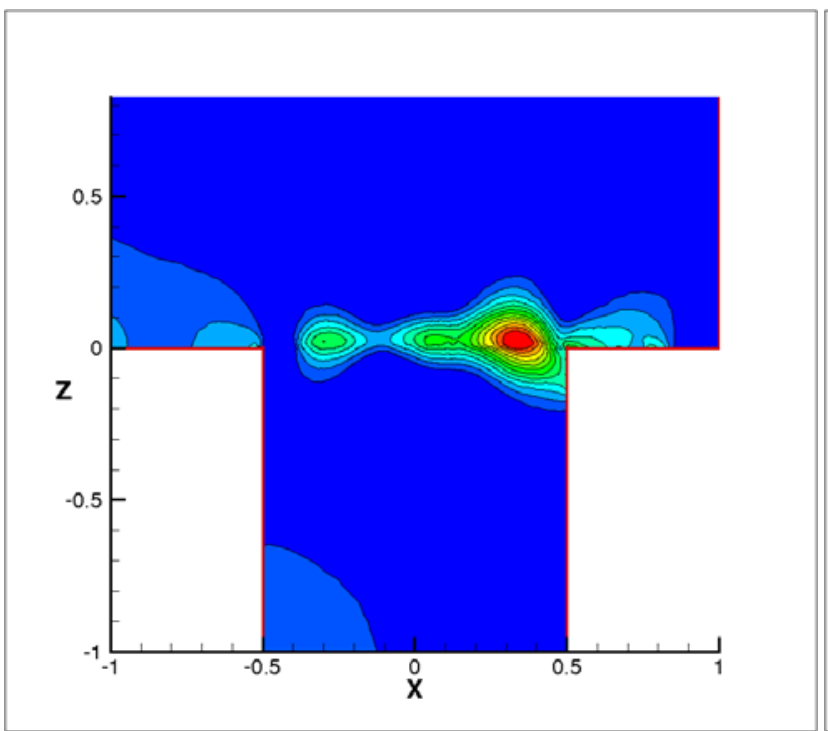

(a) KH III

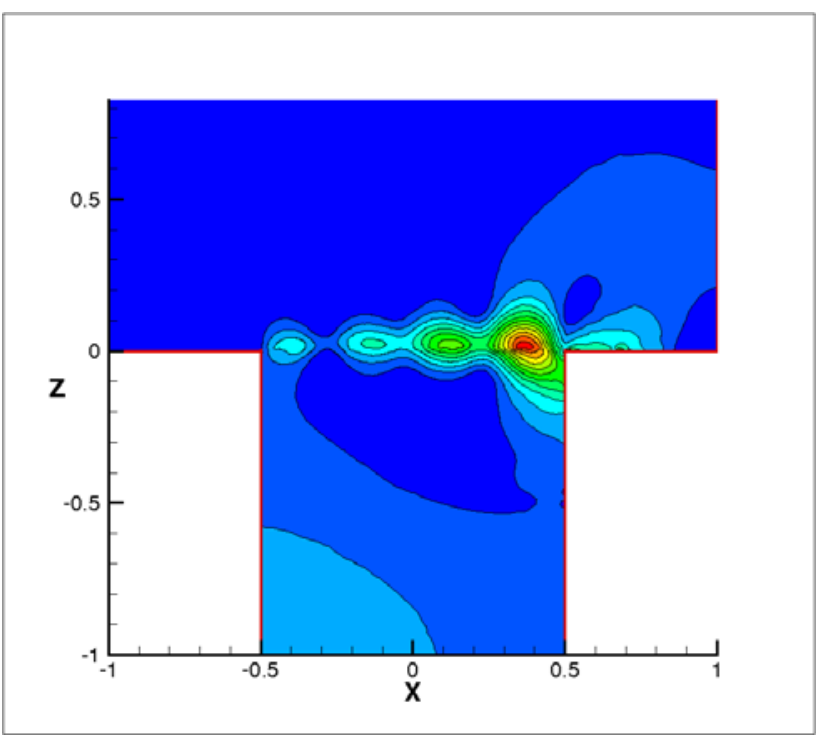

(b) KH IV

Figure 4. KH modes III and IV. Iso-values of the pressure perturbation modulus 


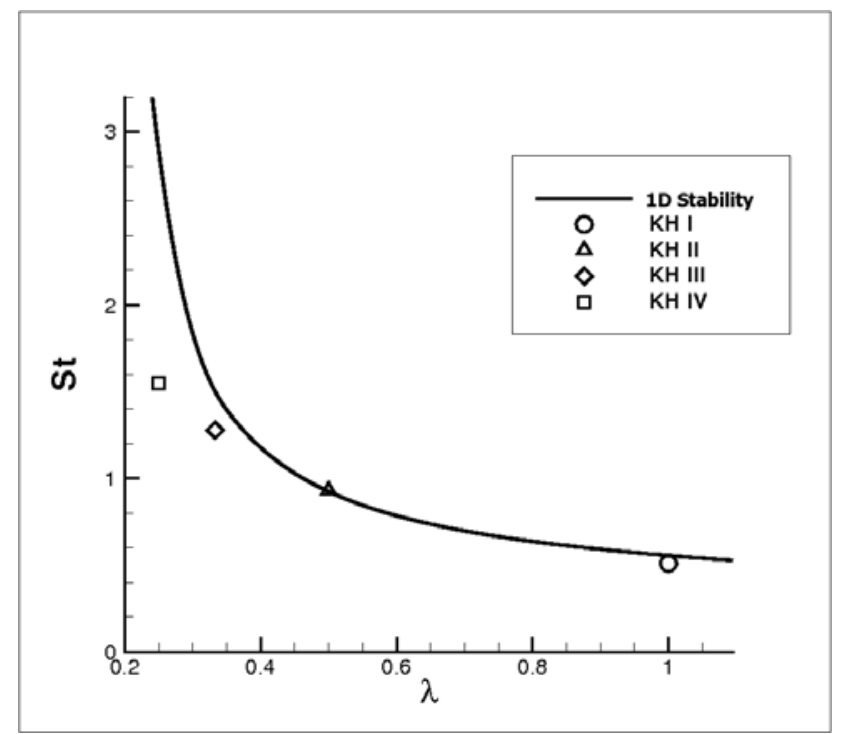

Figure 5. Comparison between the global LST and the 1D LST. 1D wave length (curve) vs the Strouhal number 2D results for $\lambda \in\{1 ; 1 / 2 ; 1 / 3 ; 1 / 4\}$ (symbols)

\section{B. Results of the unsteady part}

LES simulations provide also an unsteady flow field. Three sensors have been chosen in the median plan (figure 1). In each sensor location, a spectral density analysis is performed from the instantaneous pressure signal. Results are reported in figure 6.

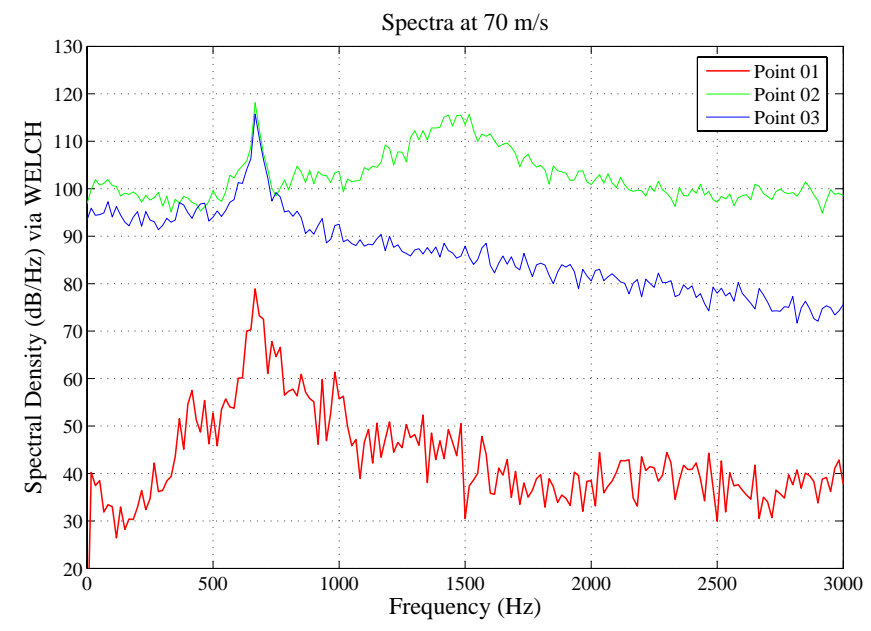

Figure 6. Spectral density for three sensors in the LES simulation

Sensor 2 is located in the shear layer which is turbulent so the spectral density is rather high regarding the other sensor signals. The most striking feature is, actually that the spectral density of sensor 2 shows a resonating frequency. This resonating frequency is $f=667 \mathrm{~Hz}$ which corresponds to a Strouhal number of $S t=0.95$. This frequency is also amplified in the two different other sensor locations. Indeed, in the bottom of the cavity, the same frequency is pointed out even if the spectral density for this sensor is obviously less powerful. Sensor 1 also detects this resonating frequency which aims at thinking that this frequency is an acoustical frequency. This frequency is also in good agreement with the acoustical frequency observed in the experiment. ${ }^{11}$ On the other hand, it must be emphasized that the present resonating mode $(f=667 \mathrm{~Hz}$ i.e. $S t=0.95)$ corresponds to the KH II mode. The frequency coincides with the frequency of an intrinsic instability mode. 


\section{Cross comparisons : LES vs LST}

Figure 7 shows the real part of the vertical velocity component $\widetilde{w}$. The plot corresponds to the KH II mode on the left hand side and to the real part of the discrete Fourier transform at the resonating frequency on the right hand side.

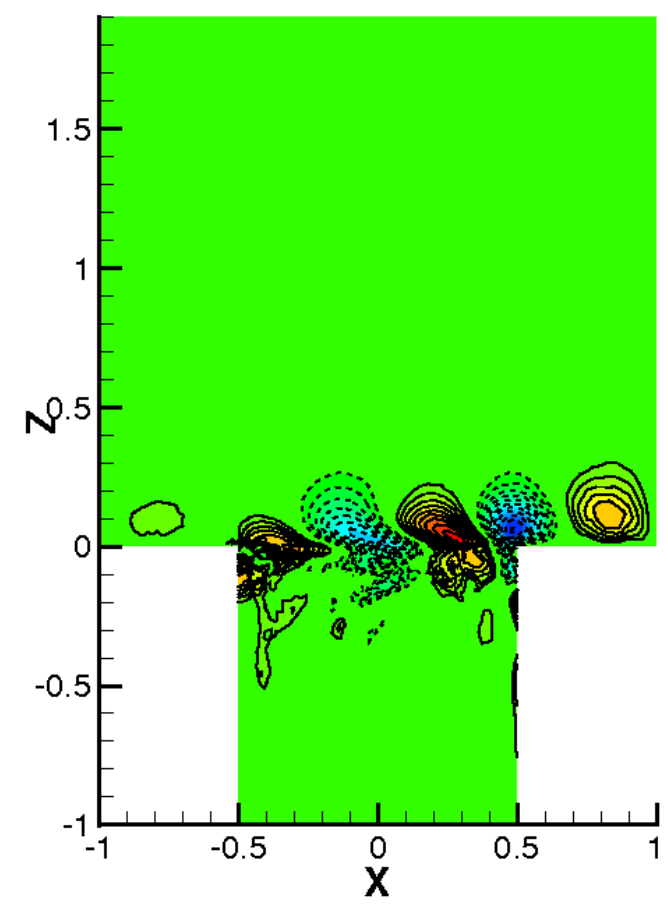

(a) LST

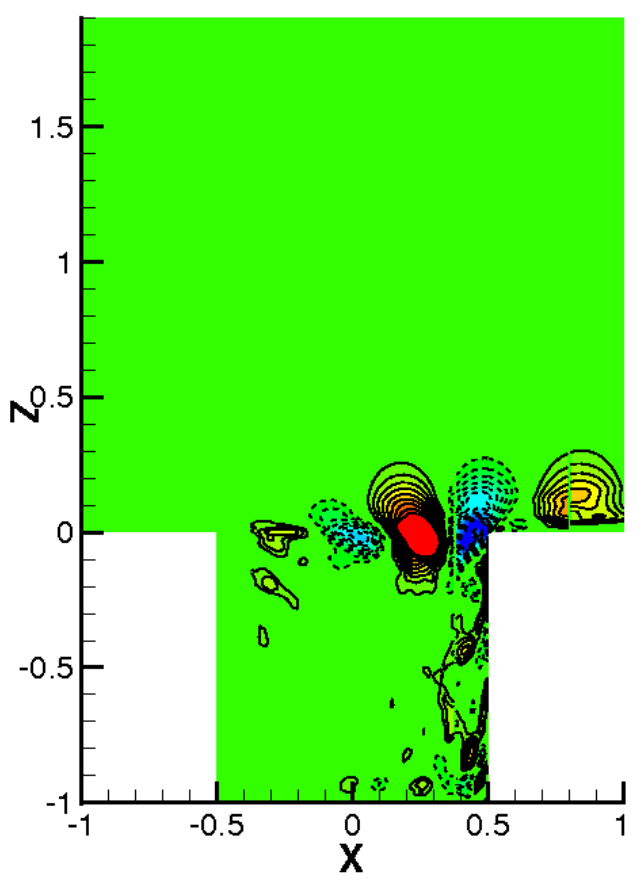

(b) LES

Figure 7. Comparison of modes shape (real part) for vertical velocity fluctuations. Kelvin-Helmholtz mode II $\left(S t_{I I}\right)(\mathrm{a})$ and discrete Fourier transform of the resonating frequency from the LES computation (b)

First, it can be noted that the normal velocity fluctuation has a particular "bean" shape. This one has been already observed. ${ }^{17}$ Other works ${ }^{7,}{ }^{18}$ show similar modes computed with DNS simulations. Secondly, the good agreement between the LES and the LST results confirms the agreement between the Strouhal number as mentioned above. Indeed, this agreement may give an explanation of the birth of acoustic phenomena. Even if the acoustic propagation mechanism is not yet detected, the origin of the acoustic wave seems to be directly linked to Kelvin-Helmholtz instability.

\section{Conclusion}

This study is a first analysis of global instabilities of a high Reynolds number and compressible flow over a cylindrical cavity using linearized inviscid equations. This paper shows the efficiency of a simple method (global instability theory) for complex flow configurations (high Reynolds number, complex geometry...). The results enable a new interpretation of the Rossiter formula, particularly, for modes I and II. Moreover, the origin of the noise is clearly pointed out : Kelvin-Helmholtz mode II seems to be the origin of the noise generation for this configuration. 


\section{Acknowledgments}

This work is a part of AEROCAV project which is supported by FRAE ("Fondation de la Recherche pour l'Aeronautique et l'Espace"). Fabien Mery's Phd is supported by DGA, the French Defence agency.

\section{References}

${ }^{1}$ M.J.Czech, J.D.Crouch, Stoker, R., Strelets, M., and Garbaruk, A., "Cavity noise generation for circular vent holes," 12th AIAA/CEAS Aeroacoustis Conference, May 2006.

${ }^{2}$ Tam, C. and Burton, D., "Sound generated by instability waves of supersonic flows," Journal of Fluid Mechanics, Vol. 138, 1984, pp. 249-271; 273-295.

${ }^{3}$ Tam, C. and Morris, P., "The radiation of sound by the instability waves of a compressible plane turbulent shear layer," Journal of Fluid Mechanics, Vol. 98(2), 1980, pp. 349-381.

${ }^{4}$ Goldstein, M., "Aeroacoustics of turbulent shear flow," Ann. Rev. Fluid. Mech., Vol. 16, 1984, pp. 263-285.

${ }^{5}$ Tatsumi, T. and Yoshimura, T., "Stability of the laminar flow in a rectangular duct." Journal of Fluid Mechanics, Vol. 212, 1990, pp. 437-449.

${ }^{6}$ Theofilis, V., "Linear instability analysis in two spatial dimensions," Athens, Greece, 1998.

${ }^{7}$ G.A.Bres and T.Colonius, "Three-dimensional linear stability analysis of cavity flow," Reno, Nevada, Janvier 2007.

${ }^{8}$ G.A.Bres and T.Colonius, "Direct numerical simulation of three-dimensional cavity flows," Roma, Italia, 2007.

${ }^{9}$ Mary, I. and Sagaut, P., "LES of a flow around an airfoil near stall," AIAA Journal, Vol. 40, 2002, pp. 1139-1145.

${ }^{10}$ Mincu, D., Mary, I., Redonnet, S., Larcheveque, L., and Dussauge, J.-P., "Numerical simulations of the unsteady flow and radiated noise over a cylindrical cavity," 14th AIAA/CEAS Aeroacoustics Conference, Vancouver, May 2008.

${ }^{11}$ Marsden, O., Jondeau, E., Souchotte, P., Bogey, C., Bailly, C., and Juve, D., "Investigation of flow features acoustic radiation of round cavity," AIAA Paper 2008-2851, may 2008.

${ }^{12}$ Rossiter, J., "Wind-tunnel experiments on the flow over rectangular cavities at subsonic and transonic speeds," Reports and memoranda n 3438, Aeronautical research council, 1964.

${ }^{13} \mathrm{Tam}$, C. and Block, P., "On the tones and pressure oscillations induced by flow over rectangular cavities," Journal of Fluid Mechanics, Vol. 89, 1978, pp. 373-399.

${ }^{14}$ Drazin, P. and Reid, W., Hydrodynamic stability, Cambridge Press, New York, 1981.

${ }^{15}$ Arnoldi, W., "The principle of minimized iteration in the solution of the matrix eigenvalue problem," Quart. Appl. Math., Vol. 9, 1951, pp. 17-29.

${ }^{16}$ Blumen, W., "Shear layer instability of an inviscid compressible fluid," Journal of Fluid Mechanics, Vol. 40, 1970, pp. 769-781.

${ }^{17}$ Rowley, C. and Colonius, T., "On self-sustained oscillations in two-dimensional compressible flow over rectangular cavities," Journal of Fluid Mechanics, Vol. 455, 2002, pp. 315-346.

${ }^{18}$ Rowley, C., Modeling, simulation and control of cavity flow oscillations, Ph.D. thesis, California Institute of Technology, 2002. 